\title{
TESTE DO CAPM CONDICIONAL DOS RETORNOS DE CARTEIRAS DOS MERCADOS BRASILEIRO, ARGENTINO E CHILENO, COMPARANDO-OS COM O MERCADO NORTE-AMERICANO
}

TEST OF THE CONDITIONAL CAPM USING RETURNS OF BRAZILIAN, ARGENTINEAN AND CHILEAN MARKETS, COMPARING THEM TO THE NORTH AMERICAN PORTFOLIO

PRUEBA DEL CAPM CONDICIONAL DE LOS RENDIMIENTOS DE CARTERAS EN LOS MERCADOS BRASILEÑO, ARGENTINO Y CHILENO EN COMPARACIÓN CON EL MERCADO NORTEAMERICANO

\section{RESUMO}

Nas últimas décadas, o modelo Capital Asset Pricing Model (CAPM) tem despertado grande interesse por parte da comunidade científica. Apesar das críticas, o aprimoramento do CAPM estático deu origem a novos modelos dinâmicos que trazem maior segurança para o investidor ao longo do ciclo de negócios. Atualmente, encontramos adaptações mais complexas do modelo CAPM, as quais nos permitem ter respostas sobre questões em finanças que por muito tempo permaneceram não solucionadas. Diante desse panorama e considerando todo o debate acerca da validade do CAPM, este trabalho tem como objetivo testar o modelo CAPM condicional de Jagannathan e Wang (1996), incorporando variáveis macroeconômicas e financeiras, para o mercado brasileiro, argentino, chileno, e norte americano.

PALAVRAS-CHAVE CAPM condicional, mercados financeiros, carteiras, modelos dinâmicos e estáticos, investimentos.

Elmo Tambosi Filho elmotf@hotmail.com

Doutor em Engenharia de Produção pelo Programa de Engenharia da Produção, Universidade Federal de Santa Catarina - Florianópolis - SC, Brasil

Fábio Gallo Garcia fabio.gallo@fgv.br

Professor da Escola de Administração de Empresas de São Paulo, Fundação Getulio Vargas - São Paulo - SP, Brasil

Joshua Onome Imoniana josh.imoniana@yahoo.com

Professor do Centro Ciências Sociais Aplicadas, Universidade Presbiteriana Mackenzie, São Paulo - SP, Brasil

Luiz Maurício Franco Moreiras luiz.moreira@bcb.gov.br

Analista do Banco Central do Brasil - São Paulo - SP, Brasil

Recebido em 08.04.2008. Aprovado em 21.12.2009

Avaliado pelo sistema double blind review. Editora Científica: Fernanda Finotti Perobelli

\begin{abstract}
Over the last few decades the Capital Asset Pricing Model (CAPM) has roused great interest in the scientific community. Despite suffering criticism, improvements in the static CAPM have given rise to new dynamic models that provide the investor with enhanced safety over the period of the business cycle. Currently, we find more complex adaptations of the CAPM, which provide us with answers to questions in finance that have long remained unsolved. Given this panorama and considering the whole debate about the feasibility of the CAPM, the objective of this work is to test the conditional Capital Asset Pricing Model of Jagannathan and Wang (1996), which incorporates macroeconomic and financial variables, for the Brazilian, Argentinian, Chilean and North American markets.

KEYWORDS Conditional CAPM, financial markets, portfolio, dynamic and static models, investiments.

RESUMEN En las últimas décadas, el CAPM (Capital Asset Pricing Model) ha despertado gran interés por parte de la comunidad científica. A pesar de las críticas, el perfeccionamiento del CAPM estático dio origen a nuevos modelos dinámicos que le proporcionan mayor seguridad al inversor a lo largo del ciclo de negocios. Actualmente, encontramos adaptaciones más complejas del CAPM, que nos permiten tener respuestas sobre cuestiones financieras que durante mucho tiempo permanecieron no solucionadas. Ante ese panorama y considerando todo el debate acerca de la validez del CAPM, este trabajo tiene como objetivo probar el CAPM condicional de Jagannathan y Wang (1996), incorporando variables macroeconómicas y financieras, para el mercado brasileño, argentino, chileno y norteamericano.
\end{abstract}

PALAVRAS CLAVE CAPM condicional, mercados financieros, carteras, modelos 


\section{INTRODUÇÃO}

Evidenciou-se nas últimas duas décadas o crescimento do número de estudos empíricos que examinam a capacidade preditiva da versão estática do CAPM. Os resultados obtidos nesses estudos mostram que o CAPM estático é incapaz de explicar razoavelmente a variação cross-sectional do retorno médio dos portfólios analisados.

Costa Jr. (1996) reforça essa ideia ao dizer que a versão original ou padrão do CAPM, de grande simplicidade, reconhece a informação de maior relevância e a utiliza de maneira facilmente compreensível. O problema reside no fato de que as hipóteses de partida dessa versão requerem um mercado em competição perfeita. Esse fato gera problemas de credibilidade em razão da falta de realismo. A resposta a essa dúvida se encontra nos testes empíricos: o importante não é o realismo das hipóteses de partida, e sim se as conclusões do modelo se ajustam à realidade.

Esse tipo de análise requer restrições sobre a distribuição dos retornos ou sobre a função utilidade dos indivíduos que nem sempre são consistentes. São alguns exemplos: a distribuição normal do retorno dos ativos, não limitada inferiormente, e a função utilidade quadrática, apresentando aversão absoluta ao risco crescente. Uma segunda restrição, a chamada crítica de Roll (1977), ataca o CAPM pela sua incapacidade de ser testado empiricamente. A impossibilidade de observar a carteira de mercado e a crítica de que a real hipótese verificada nos testes propostos para o CAPM não é a hipótese de Sharpe, mas a hipótese de que a carteira de mercado é eficiente, levaram à conclusão de que nenhum teste já feito realmente foi capaz de testar a teoria do CAPM.

Fama e French (1992), críticos ferrenhos do CAPM, fizeram testes multivariáveis (regressões múltiplas) e encontraram duas variáveis que explicam a maior parte das variações de cross-section dos retornos médios: o índice valor contábil/valor de mercado tem uma relação positiva com os retornos das ações enquanto a variável tamanho tem uma relação negativa bastante significativa, sendo que o beta teve pouca significância nesse teste.

Embora reconheçamos que o CAPM e suas versões estáticas foram e são de grande importância em finanças, nos dias de hoje encontramos adaptações mais complexas do modelo CAPM que nos permitem vislumbrar respostas a questões que ainda permanecem sem resposta em finanças.

Diante desse panorama e considerando toda a grande discussão acerca da validade do CAPM, este trabalho procura apresentar as vantagens dos modelos condicionais ou dinâmicos (modelos que incorporam variâncias e covariâncias que se alteram ao longo do tempo), em relação ao modelo estático.
Para constatar tais fatos estudar-se-ão os testes dos modelos condicionais (beta variando ao longo do tempo) que não são comumente estudados na literatura. Esses testes são convenientes para incorporar variâncias e covariâncias que se alteram ao longo do tempo. Dentre os testes dos modelos condicionais, destacamos o de Jagannathan e Wang (1996).

Bonomo (2002) destaca alguns importantes estudos do CAPM condicional, entre eles o proposto por Bodurtha e Mark (1991), onde o beta de uma carteira de ativos é definido como a covariância condicional do erro de previsão do retorno da carteira e o erro de previsão do retorno do mercado. Esses modelos possuem vários coeficientes betas, enquanto o CAPM original possui apenas um.

O presente estudo encontra-se estruturado em outras quatro seções, além desta Introdução: a segunda seção traz algumas breves considerações sobre o CAPM condicional. A terceira traz detalhes do modelo CAPM condicional para o Brasil, a Argentina e o Chile. A quarta mostra os resultados obtidos para o mercado brasileiro, argentino, chileno e norte-americano. E, finalmente, a quinta seção mostra as conclusões finais.

\section{CONSIDERAÇÕES INICIAIS ACERCA DO CAPM CONDICIONAL}

Ribenboim (2002) reforça a ideia de que o CAPM condicional permite que o prêmio de risco de um ativo possa mudar a partir da variação de um ou mais dos seguintes componentes: a variância condicional do retorno da carteira de mercado, a covariância condicional entre o retorno do ativo e o retorno da carteira de mercado e o prêmio de risco da carteira de mercado. Quando a covariância condicional entre cada ativo e o mercado é proporcional à variância condicional, recaímos no CAPM não condicional.

O CAPM condicional proposto por Jagannathan e Wang (1996) usa o subscrito $t$ para indicar o período de tempo relevante. $R_{i t}$ denota o retorno do ativo $i$ no período $t$, enquanto $R_{m t}$ representa o retorno da riqueza agregada da carteira de todos os ativos da economia no período $t$.

$$
E\left[R_{i t} \mid I_{t-1}\right]=\gamma_{0 t-1}{ }^{1}+\gamma_{1 t-1} \beta_{i t-1}
$$

Onde $I_{t-1}$ é o nível de informação dos investidores no final do período $t-1$ e $\beta_{i t-1}=$ é o beta condicional do ativo $i$ no período $t-1$ definido como:

$$
\beta_{i t-1}=\operatorname{Cov}\left(R_{i t}, R_{m t} \mid I_{t-1}\right) / \operatorname{Var}\left(R_{m t \mid} \mid I_{t-1}\right)
$$

$\gamma_{0 t-1}$ é o retorno esperado condicional sobre a carteira com 
beta igual a zero, $\gamma_{1 t-1}$ é o prêmio do risco de mercado condicional. Jagannathan e Wang (1996), para explicar a variação cross-sectional do retorno esperado não condicional em diferentes ativos, colocam a expectativa não condicional em ambos os lados da equação (2), encontrando a equação abaixo:

$$
E\left[R_{i t}\right]=\gamma_{0}+\gamma_{1} \bar{\beta}_{i}+\operatorname{Cov}\left(\gamma_{1 t-1} \beta_{i t-1}\right)
$$

Onde $\gamma_{0}=E\left[\gamma_{0 t-1}\right] \gamma_{1}=E\left[\gamma_{1 t-1}\right] \bar{\beta}_{i}=E\left[\beta_{i t-1}\right]$

Aqui, $\gamma_{1}$ é o prêmio esperado do risco de mercado, e $\bar{\beta}_{i}$ é o beta esperado. Se a covariância entre o beta condicional $\beta_{i t-1}$ dos ativos $i$ e o prêmio do risco de mercado condicional for zero ou função linear do beta esperado para uma escolha arbitrária de ativos $i$, a equação (3) lembra o CAPM estático, onde o retorno esperado é função linear do beta esperado. De qualquer modo, geralmente, o prêmio do risco de mercado condicional e os betas condicionais são correlacionados.

Entretanto, os trabalhos de Kein e Stambaugh (1986), Mayers (1972), Breem, Glosten e Jagannathan (1989), Fama e French (1989) e Ferson e Harvey (1991) mostram que o prêmio esperado do risco dos ativos e os betas condicionais não são constantes. Portanto, geralmente, $o$ último termo da equação (3) não é zero e o retorno esperado não condicional não é função linear do beta esperado.

Esse fato motivou Jagannathan e Wang (1996) a decompor o beta condicional do ativo $i \mathrm{em}$ dois componentes ortogonais para projetar o beta condicional do prêmio de risco do mercado. Para cada ativo $i$, define-se a sensibilidade do beta do prêmio de mercado (denotado por $\vartheta_{i}$ ) e o beta residual (denotado por $\eta_{i t-1}$ ):

$$
\begin{aligned}
& \text { Onde } \vartheta_{i}=\operatorname{Cov}\left(\beta_{i t-1}, \gamma_{1 t-1}\right) / \operatorname{Var}\left(\gamma_{1 t-1}\right) \\
& \eta_{i t-1}=\beta_{i t-1},-\bar{\beta}_{i}-\vartheta_{i}\left(\gamma_{1 t-1}-\gamma_{1}\right)
\end{aligned}
$$

$\mathrm{Na}$ expressão acima, o $\vartheta_{i}$ mede a sensibilidade do beta condicional para o prêmio de risco de mercado. Para cada ativo $i$ tem-se

$$
\beta_{i t-1}=\bar{\beta}_{i}+\vartheta_{i}\left(\gamma_{1 t-1}-\gamma_{1}\right)+\eta_{i t-1}
$$

$$
\mathrm{E}\left[\eta_{i t-1}\right]=0
$$

$$
\mathrm{E}\left[\eta_{i t-1} \gamma_{i t-1}\right]=0
$$

$\mathrm{Na}$ equação (6), cada beta condicional é decomposto em três partes ortogonais: a primeira corresponde ao beta esperado, que é a constante; a segunda corresponde à variável randômica, que é perfeitamente correlacionada com o prêmio de risco do mercado; a última refere-se à média zero que não é correlacionada com o prêmio de risco do mercado.

Mayers (1972) mostra que o capital humano tem relevante participação no total do capital investido na economia. Seguindo essa sugestão Jagannathan e Wang (1996) incluíram, na proxy do retorno de mercado, o retorno do capital humano através da introdução da taxa de crescimento da renda do trabalho, que é detalhada a seguir:

$$
R_{m t}=\phi_{0}+\phi_{v w} R_{t}^{v w}
$$

Onde $R_{t}^{v w}$ denota o retorno do value weighted stock index portfolio. Assume-se implicitamente que o retorno de mercado é função linear do índice de mercado composto por ações da NYSE, AMEX e NASDAQ. Existem algumas constantes $\phi_{0}$ e $\phi_{v w}$ no modelo. Sendo $W$ a taxa de crescimento per capita da renda do trabalho e $L_{t}$ é a taxa de retorno esperada da renda do trabalho.

A razão da troca da riqueza é então dada por:

$$
R_{t}^{\text {labor }}=L_{t}-L_{t-1} / L_{t-1}
$$

São admitidas ambas as suposições: parte da taxa de crescimento da renda do trabalho e parte dos retornos dos ativos não são importantes. O termo adicionado sobre a taxa de crescimento da renda do trabalho será muito pequeno.

Jagannathan e Wang (1996) assumem que o retorno do capital humano é uma função linear da taxa de crescimento per capita da renda do trabalho. Assim, a medida do retorno do capital humano é incorporada ao modelo, assumindo que o retorno de mercado é uma função linear do $R_{t}^{v w}$ e $R_{t}^{\text {labor }}$.

A seguir, Jagannathan e Wang (1996) apresentam outra suposição que tem por finalidade introduzir algumas constantes no modelo com a finalidade de transformar o modelo estático em condicional.

Existem algumas constantes $\phi_{0}, \phi_{v w}$, $\phi_{\text {labor }}$ no modelo:

$$
R_{m t}=\phi_{0}+\phi_{v w} R_{t}^{v w}+\phi_{\text {labor }} R_{t}^{\text {labor }}
$$

O labor-beta é definido como:

$$
\beta_{i}^{\text {labor }}=\operatorname{Cov}\left(R_{i t}, R_{t}^{\text {labor }}\right) / \operatorname{Var}\left(R_{t}^{\text {labor }}\right)
$$

Nas suposições, assume-se que o retorno esperado não condicional de algum ativo é função linear do beta do prêmio e do beta labor. 
A seguir, Jagannathan e Wang (1996) apresentam a relação de linearidade entre o retorno esperado não condicional e as demais variáveis. Contudo, presume-se que $\beta_{i}^{\gamma}$ não é função linear de $\beta_{i}$ e essas suposições são satisfatórias e permitem admitir a condicionalidade do modelo, levando em conta a existência de algumas constantes $c_{0}, c_{w}, c_{\text {prem }}$ e $c_{\text {labor }}$ que foram incluídas na equação a seguir:

$$
E\left[R_{i t}\right]=c_{0}+c_{v w} \beta_{i}^{v w}+c_{\text {prem }} \beta_{i}^{\text {prem }}+c_{\text {labor }} \beta_{i}^{\text {labor }}
$$

Para todos ativos $i$.

Com o objetivo de melhorar a proxy, Jagannathan e Wang (1996) seguiram Mayers (1972) e incluíram em seu modelo o retorno do capital humano. Quando o capital humano é também incluído na carteira de mercado, o modelo não condicional implícito no CAPM condicional é capaz de explicar mais de $50 \%$ da variação cross-sectional do retorno médio. Além disso, os testes estatísticos realizados pelos autores citados falharam na rejeição do modelo.

No presente trabalho, a base do estudo empírico será justamente o modelo de Jagannathan e Wang (1996) denominado Premium Labor Model.

\section{O MODELO CAPM CONDICIONAL PARA 0 BRASIL, A ARGENTINA E 0 CHILE}

As variáveis utilizadas como proxy para o capital humano são especificações comumente usadas em estudos empíricos do CAPM estático e condicional. Portanto, testes do CAPM baseados nessas especificações podem ser interpretados através de duas hipóteses: a primeira onde o CAPM estático de Sharpe se apoia e a segunda onde o retorno da carteira é função linear do retorno do índice de mercado de ações com betas condicionais. Consequentemente, os resultados podem ter várias interpretações, não ferindo a proposição do modelo de Sharpe.

Particularmente, as razões das rejeições empíricas da equação podem estar relacionadas com as limitações do CAPM estático ou, por outro lado, é possível que o CAPM estático seja aceito, mas que o retorno do índice de mercado de ações seja uma proxy pobre para o retorno da riqueza agregada. Nesse ponto, o CAPM condicional com a proxy para o capital humano procura responder de melhor maneira essa questão. Ribenboim (2002) mostra que alguns testes existentes na literatura demonstram que a utilização de uma proxy de mercado que contenha grande parte do volume transacionado, como é o caso do Ibovespa, não ocasiona perdas significativas no teste do modelo. A utilização dos coeficientes do mercado acionário brasileiro se justifica, já que em estudos empíricos do CAPM é comumente considerado que o retorno da riqueza da carteira das ações negociadas nos mercados é uma boa proxy para o retorno da riqueza agregada da carteira.

As variáveis selecionadas a priori, que farão parte do modelo CAPM condicional para o Brasil, referem-se a carteiras construídas através dos retornos mensais de ações cotadas na Bolsa de Valores de São Paulo (Bovespa), PIB de mercado e, para o prêmio, o spread entre a taxa de operações de depósitos interfinanceiros (DI), divulgadas pela Central de Custódia e Liquidação de Títulos Privados (CETIP) e a taxa de juros (Selic), que tem a finalidade de ser um previsor para as variações dos ciclos de negócios. Para o mercado argentino, o prêmio é representado pelo spread entre a taxa de juros sobre empréstimos ao setor privado e a taxa de juros básica da economia. Já o capital humano é representado pelo PIB argentino. A proxy de mercado será o índice Merval.

Utilizando a metodologia descrita acima, foram criadas sete carteiras para o mercado brasileiro, contendo cinco ações do Ibovespa no período de jan/94 até dez/02, totalizando 108 observações. Para o mercado argentino, foram criadas cinco carteiras, contendo cinco ações da Bolsa de Valores da Argentina (Merval). E finalmente, para o mercado chileno, foram utilizadas 25 ações divididas em cinco carteiras. A escolha das ações deve-se puramente a uma questão de liquidez. Todas as ações receberam o mesmo peso dentro de cada portfólio.

Para o Chile foi também utilizado o PIB mensal e para o prêmio o spread entre a taxa de juros de linha de crédito para o setor secundário e a taxa de juros básica da economia. Os retornos de mercado para o Chile foram retirados do índice IPSE. Os dados foram coletados no Banco Central do Brasil, Banco Central da Argentina, Banco Central do Chile e Economática.

De maneira similar, Jagannathan e Wang (1996) utilizaram os retornos de todas as ações da NYSE e AMEX e montaram 100 carteiras em função da variável tamanho, com retornos mensais de julho de 1963 a dezembro de 1990, totalizando 330 observações. Para cada carteira calcularam-se regressões entre as ações que compõem as carteiras e o índice de mercado (NYSE e AMEX).

As regressões são estimadas usando-se a metodologia de Fama e MacBeth (1974). Os autores elaboraram sete carteiras contendo ações listadas na Bovespa e cinco carteiras listadas na Merval que foram analisadas no período de 1994 até 2002. Posteriormente, estimamos 
o beta de cada uma das carteiras, relacionando os retornos mensais do índice de mercado para o período de 1994 até 1998. Utilizamos os betas de cada uma das carteiras do período anterior para prever os retornos mensais das carteiras do período subsequente de 1999 até 2002. O processo de estimação dos betas do mercado repetiu-se seis vezes até a obtenção total de 108 estimações, que foi de janeiro de 1994 até dezembro de 2002. Ainda, em relação ao beta, foi estimado para cada ação, usando 24 e 60 meses do retorno passado das ações das bolsas analisadas. Seguindo o procedimento realizado por Fama e French (1992), foram criadas sete carteiras para o Brasil, cinco para a Argentina e cinco para o Chile e computou-se o retorno de cada uma delas para os 12 meses seguintes. Foi repetido esse procedimento para cada ano analisado.

Criou-se uma série temporal do retorno mensal para cada uma das sete carteiras do Brasil, das cinco da Argentina e das cinco chilenas. O modelo para os momentos é estimado usando-se o método generalizado dos momentos.

Utilizou-se o valor médio de cada um dos coeficientes para determinar a sua significância. As carteiras foram refeitas anualmente.

De acordo com Fama e MacBeth (1974), esses portfólios eram rebalanceados período por período até que a estimação dos betas atingisse o total de estimações de todo o período analisado. Todas as ações receberam o mesmo peso dentro de cada carteira.

Já a escolha das ações se deu levando-se em conta o critério da liquidez e utilizando apenas ações sobreviventes, ou seja, ações de empresas que permaneceram cotadas na Bolsa de Valores de São Paulo, na Bolsa de Valores da Argentina e na Bolsa de Valores do Chile durante todo o período de análise do estudo.

Fama (1988) mostra que essa construção, apesar de arbitrária, é comum na literatura de testes empíricos. Apesar de Fama e French (1992) e Jagannathan e Wang (1996) não usarem ações de bancos em suas amostras, optou-se pelo uso dessas ações, já que testes de versões do modelo CAPM no Brasil já utilizam essas ações, como é o caso do estudo de Ribenboim (2002).

Uma observação que confronta a revisão teórica com a pesquisa diz respeito à escolha de um índice brasileiro, argentino e chileno de ações, como a carteira de mercado. Isso implica assumirmos implicitamente que o mercado é segmentado. Bonomo (2002) mostra que, se o mercado é segmentado, um grande número de investidores diversificará internacionalmente sua carteira e o mercado se moverá no sentido de uma integração, e os retornos esperados no Brasil, na Argentina e no Chile serão bem descritos pela exposição dos países ao risco mundial.

\section{RESULTADOS OBTIDOS PARA OS MERCADOS BRASILEIRO, ARGENTINO E CHILENO EM COMPARAÇÃO COM $O$ MERCADO NORTE- AMERICANO}

A seguir, serão apresentadas as tabelas mostrando os resultados do modelo CAPM estático sem capital humano para os países analisados.

Os resultados presentes nas Tabelas 1, 2, 3 e 4 mostram que o $t$ value para o $C_{\text {ibov }}$ é 0,17 . O R2 da regressão é de somente $8,49 \%$. Isto quer dizer que a variação crosssectional média dos retornos ainda não é bem explicada quando utilizamos o CAPM estático sem a inclusão do PIB de mercado no caso brasileiro.

Os resultados presentes na Tabela 2 mostram que o $t$ value para o $C_{\text {MERVAL }}$ é $-1,34$ que corresponde a um $p$ value de $54 \%$. O R2 da regressão é de somente 5,40\%. Isto quer dizer que a variação cross-sectional média dos retornos não é bem explicada quando utilizamos o CAPM estático sem a inclusão do capital humano para o mercado argentino.

Os resultados presentes na Tabela 3 mostram que o $t$ value para o $\mathrm{C}_{\mathrm{IPSE}}$ é 1,34 . O R2 da regressão é de somente $6,30 \%$. Isto quer dizer que a variação cross-sectional média dos retornos para o mercado chileno ainda não é bem explicada quando utilizamos o CAPM estático sem a inclusão do PIB no caso chileno.

O modelo de correção de erros para estimação não é significativo. Após a correção dos erros, que trata o termo de erro do modelo, podemos usar esse termo para ligar o comportamento das variáveis no curto prazo com seu valor no longo prazo, ou seja, é um meio de reconciliar o comportamento no curto prazo de uma variável com seu comportamento no longo prazo. $\mathrm{O}_{\mathrm{ibov}}$ não é significativamente diferente de zero. Quando é introduzida a variável size no modelo, encontramos para o $C_{\text {size }}$ um $t$-value de 3,29 e o $R 2$ sobe para $42,10 \%$.

Apesar do aumento do R2 e do fato de o modelo não apresentar nenhuma mudança significativa após a correção dos erros, o modelo parece inconsistente (porque mesmo após a inclusão da variável size para o mercado brasileiro, este parece não ser influenciado por aquela, uma vez que o modelo estático não capta o efeito dessa variável). A análise do mercado brasileiro parece caminhar para uma conclusão semelhante à dos mercados norteamericano, argentino e chileno. 
A seguir, serão apresentadas as tabelas que mostram os resultados do modelo CAPM condicional sem capital humano para os países analisados.
Os resultados presentes na Tabela 5 mostram que o valor estimado para o $C_{\text {premio }}$, não é significativamente diferente de zero. O t-value para $C_{\text {premio }}$ é de $-0,52$. O R2 é de

Tabela 1 - CAPM estático sem capital humano (Brasil)

\begin{tabular}{|c|c|c|c|c|c|c|}
\hline COEFICIENTES & $\mathrm{C}_{0}$ & $\mathrm{C}_{\mathrm{IBOV}}$ & $\mathbf{C}_{\text {PREMIO }}$ & $\mathrm{C}_{\text {PIB MER }}$ & $\mathrm{C}_{\text {SIZE }}$ & R-SQUARE \\
\hline Estimate & $-2,67$ & 1,25 & & & & 8,49 \\
\hline t-value & $-0,76$ & 0,17 & & & & \\
\hline$p$-value & 0,00 & 0,00 & & & & \\
\hline Correção-t & $-0,19$ & 0,08 & & & & \\
\hline Correção-p & 0,00 & 0,00 & & & & \\
\hline Estimate & $-0,88$ & 0,44 & & & 0,61 & 42,10 \\
\hline t-value & $-0,24$ & 0,06 & & & 3,29 & \\
\hline$p$-value & 0,00 & 0,00 & & & 0,00 & \\
\hline Correção-t & $-0,14$ & 0,05 & & & 2,97 & \\
\hline Correção-p & 0,00 & 0,00 & & & 0,00 & \\
\hline
\end{tabular}

Nota: As regressões do modelo são estimadas usando-se a metodologia de Fama e MacBeth (1974). 0 modelo foi estimado usando-se o método generalizado dos momentos. Através da correção dos erros, verificamos se a variância residual afeta o preço dos ativos ou a taxa de retorno esperada e, com base nos resultados, não existe uma indicação de que os ativos com variância residual maior que a média produzam taxa de retorno maior que a média num período futuro. Foram construídos sete portfólios com cinco ações em cada um deles. O período testado vai de janeiro de 1994 até dezembro de 2002. Para o mercado brasileiro, o prêmio é representado pelo spread entre a taxa da CETIP e a taxa SELIC. Já o capital humano é representado pelo PIB de mercado. A proxy de mercado será o Ibovespa. A equação que está sendo estimada para o mercado brasileiro é a seguinte:

$E\left[R_{i t}\right]=c_{0}+c_{\text {size }} \log \left(M E_{i}\right)+c_{i b o v} \beta+c_{\text {premio }} \beta+c_{\text {pib.mer }} \beta$

\section{Tabela 2 - CAPM estático sem capital humano (Argentina)}

\begin{tabular}{|c|c|c|c|c|c|c|}
\hline COEFICIENTES & $\mathrm{C}_{0}$ & $\mathbf{C}_{\text {MERVAL }}$ & $\mathrm{C}_{\text {PREMIO }}$ & $\mathrm{C}_{\mathrm{PIB}}$ & $\mathrm{C}_{\text {SIZE }}$ & R-SQUARE \\
\hline Estimate & 0,28 & 1,17 & & & & 5,40 \\
\hline t-value & 1,45 & $-1,34$ & & & & \\
\hline$p$-value & 0,00 & 54,00 & & & & \\
\hline Correção-t & 2,18 & $-1,24$ & & & & \\
\hline Correção-p & 0,00 & 55,00 & & & & \\
\hline Estimate & 0,80 & 1,67 & & & $-1,74$ & 46,60 \\
\hline t-value & 2,20 & 2,13 & & & $-3,45$ & \\
\hline$p$-value & 0,00 & 45,13 & & & 0,70 & \\
\hline Correção-t & 2,13 & 1,12 & & & $-2,30$ & \\
\hline Correção-p & 0,00 & 47,70 & & & 2,17 & \\
\hline
\end{tabular}

Nota: As regressões do modelo são estimadas usando-se a metodologia de Fama e MacBeth (1974). 0 modelo foi estimado usando-se o método generalizado dos momentos. Através da correção dos erros, verificamos se a variância residual afeta o preço dos ativos ou a taxa de retorno esperada e, com base nos resultados, não existe uma indicação de que os ativos com variância residual maior que a média produzam taxa de retorno maior que a média num período futuro. Foram construídos cinco portfólios com cinco ações em cada um deles. O período testado vai de janeiro de 1994 até dezembro de 2002. Para o mercado argentino, o prêmio é representado pelo spread entre a taxa de juros sobre empréstimos ao setor privado e a taxa de juros básica da economia. Já o capital humano é representado pelo PIB argentino. A proxy de mercado será o índice Merval. A equação que está sendo estimada para o mercado argentino é a seguinte: $E\left[R_{i t}\right]=c_{0}+c_{\text {size }} \log \left(M E_{i}\right)+c_{\text {merval }} \beta+c_{\text {premio }} \beta+c_{\text {pib }} \beta$ 
somente $9,82 \%$. Nota-se que o $R 2$ é similar ao resultado encontrado no modelo anterior.

Já os resultados presentes na Tabela 6 mostram que o valor estimado para o $C_{\text {premio, }}$, usando-se a metodologia de Fama-MacBeth, é significativamente diferente de zero. O t-value para $C_{\text {premio }}$ é de 2,70 com o p-value de $0,30 \%$. O R2 é de 15,50\%. Nota-se um substancial aumento do R2 se comparado com o modelo visto anteriormente. Quando se introduz o modelo de correção de erros, o $t$-value para $C_{\text {premio }}$ é de 1,78 e o $p$-value vai para $0,40 \%$.
Quando a variável size é adicionada ao modelo, o t-value para $C_{\text {size }}$ é de -2,03 com o p-value de 3,45\%. Quando se introduz o modelo de correção de erros, o t-value para o $C_{\text {size }}$ cai para $-2,30$ e o $p$-value vai para $4,70 \%$. O R2 sobe para $52,70 \%$.

Quando se introduz o modelo de correção de erros, o $t$-value para $C_{\text {premio }}$ é de -0,36. Quando a variável size é adicionada ao modelo, o t-value para $C_{\text {size }}$ é de 3,03. Quando se introduz o modelo de correção de erros, o $t$-value para o $C_{\text {size }}$ cai para 2,68 , e o $R 2$ sobe para $42,90 \%$.

\section{Tabela 3 - CAPM estático sem capital humano (Chile)}

\begin{tabular}{|c|c|c|c|c|c|c|}
\hline COEFICIENTES & $\mathrm{C}_{0}$ & $\mathrm{C}_{\text {IPSE }}$ & $\mathrm{C}_{\text {PREMIO }}$ & $\mathrm{C}_{\mathrm{PIB}}$ & $\mathrm{C}_{\mathrm{SIZE}}$ & R-SQUARE \\
\hline Estimate & $-3,34$ & 2,56 & & & & 6,30 \\
\hline t-value & $-1,84$ & 1,34 & & & & \\
\hline$p$-value & 0,00 & 0,00 & & & & \\
\hline Correção-t & $-2,45$ & 0,89 & & & & \\
\hline Correção-p & 0,00 & 0,00 & & & & \\
\hline Estimate & $-1,93$ & 1,23 & & & 1,84 & 39,36 \\
\hline t-value & $-1,38$ & 1,78 & & & 4,72 & \\
\hline$p$-value & 0,00 & 0,00 & & & 0,00 & \\
\hline Correção-t & $-1,12$ & 1,74 & & & 3,45 & \\
\hline Correção-p & 0,00 & 0,00 & & & 0,00 & \\
\hline
\end{tabular}

Nota: As regressões do modelo são estimadas usando-se a metodologia de Fama e MacBeth (1974). 0 modelo foi estimado usando-se o método generalizado dos momentos. Através da correção dos erros, verificamos se a variância residual afeta o preço dos ativos ou a taxa de retorno esperada e, com base nos resultados, não existe uma indicação de que os ativos com variância residual maior que a média produzam taxa de retorno maior que a média num período futuro. Foram construídas cinco carteiras com cinco ações em cada uma delas. O período testado vai de janeiro de 1994 até dezembro de 2002.

$E\left[R_{i t}\right]=c_{0}+c_{\text {size }} \log \left(M E_{i}\right)+c_{I P S E} \beta+c_{\text {premio }} \beta+c_{\text {pib }} \beta$

Tabela 4 - CAPM estático sem capital humano (EUA)

\begin{tabular}{|l|c|c|c|c|c|c|}
\hline \multicolumn{1}{|c|}{ COEFICIENTES } & $\mathrm{C}_{0}$ & $\mathrm{C}_{\mathrm{W}}$ & $\mathrm{C}_{\text {PREMIO }}$ & $\mathrm{C}_{\text {PIB }}$ & $\mathrm{C}_{\text {SIIE }}$ & R-SQUARE \\
\hline Estimate & 1,24 & $-0,1$ & & & \\
\hline t-value & 5,17 & $-0,28$ & & & \\
\hline p-value & 0 & 78 & & & \\
\hline Correção-t & 5,16 & $-0,28$ & & & \\
\hline Correção-p & 0 & 78,01 & & & \\
\hline Estimate & 2,08 & $-0,32$ & & $-0,11$ & 57,56 \\
\hline t-value & 5,79 & $-0,94$ & & $-2,3$ & \\
\hline p-value & 0 & 34,54 & & 2,14 & \\
\hline Correção-t & 5,77 & $-0,94$ & & $-2,3$ & \\
\hline Correção-p & 0 & 34,6 & & 2,17 & \\
\hline
\end{tabular}


Verifica-se na Tabela 7 que o valor do $R 2$ foi de $40,24 \%$ (muito próximo do valor encontrado no CAPM estático), ademais o valor estimado para a variável $\mathrm{C}_{\text {premio }}$, depois de corrigidos os erros, tornou-se significativamente diferente de zero, fatos estes que podem ser explicados pela não inclusão do PIB. Assim mesmo, o modelo condicional pare-

\section{Tabela 5 - CAPM condicional sem capital humano (Brasil)}

\begin{tabular}{|l|c|c|c|c|c|c|}
\hline \multicolumn{1}{|c|}{ COEFICIENTES } & $\mathrm{C}_{0}$ & $\mathrm{C}_{\text {IBOV }}$ & $\mathrm{C}_{\text {PREMIO }}$ & $\mathrm{C}_{\text {PIB MER }}$ & $\mathrm{C}_{\text {SIZE }}$ & R-SQUARE \\
\hline Estimate & $-2,67$ & $-1,09$ & $-4,74$ & & \\
\hline t-value & $-0,74$ & $-0,05$ & $-0,52$ & & \\
\hline p-value & 0,00 & 0,00 & 0,00 & & \\
\hline Correção-t & $-0,17$ & $-0,02$ & $-0,36$ & & \\
\hline Correção-p & 0,00 & 0,01 & 0,00 & & \\
\hline Estimate & $-0,87$ & 0,56 & 0,53 & & \\
\hline t-value & $-0,20$ & 0,02 & 0,33 & & \\
\hline p-value & 0,00 & 0,08 & 0,32 & & 32,90 \\
\hline Correção-t & $-0,14$ & 0,01 & 0,16 & & 0,00 & \\
\hline Correção-p & 0,00 & 0,05 & 0,51 & & 2,68 & \\
\hline
\end{tabular}

Nota: As regressões do modelo são estimadas usando-se a metodologia de Fama e MacBeth (1974). 0 modelo foi estimado usando-se o método generalizado dos momentos. Através da correção dos erros, verificamos se a variância residual afeta o preço dos ativos ou a taxa de retorno esperada e, com base nos resultados, não existe uma indicação de que os ativos com variância residual maior que a média produzam taxa de retorno maior que a média num período futuro. Foram construídos sete portfólios com cinco ações em cada um deles. O período testado vai de janeiro de 1994 até dezembro de 2002. Para o mercado brasileiro, o prêmio é representado pelo spread entre a taxa da CETIP e a taxa SELIC. Já o capital humano é representado pelo PIB de mercado. A proxy de mercado será o Ibovespa. A equação que está sendo estimada para o mercado brasileiro é a seguinte:

$E\left[R_{i t}\right]=c_{0}+c_{\text {size }} \log \left(M E_{i}\right)+c_{i b o v} \beta+c_{\text {premio }} \beta+c_{\text {pib.mer }} \beta$

Tabela 6 - CAPM condicional sem capital humano (Argentina)

\begin{tabular}{|l|c|c|c|c|c|c|}
\hline \multicolumn{1}{|c|}{ COEFICIENTES } & $\mathrm{C}_{0}$ & $\mathrm{C}_{\text {MERVAL }}$ & $\mathrm{C}_{\text {PREMIO }}$ & $\mathrm{C}_{\text {PIB }}$ & $\mathrm{C}_{\text {SIIE }}$ & R-SQUARE \\
\hline Estimate & 1,56 & 1,31 & 1,46 & & \\
\hline t-value & 1,77 & 1,83 & 2,70 & & \\
\hline p-value & 0,30 & 20,30 & 0,30 & & \\
\hline Correção-t & 1,13 & 1,34 & 1,78 & & \\
\hline Correção-p & 4,50 & 28,13 & 0,40 & & \\
\hline Estimate & 0,85 & 0,70 & 0,80 & & $-1,20$ & 52,70 \\
\hline t-value & 2,90 & $-2,30$ & 1,20 & & $-2,03$ & \\
\hline p-value & 0,00 & 18,19 & 0,80 & & 3,45 & \\
\hline Correção-t & 2,50 & $-0,80$ & 1,10 & & $-2,30$ & \\
\hline Correção-p & 0,1 & 14,53 & 0,96 & & 4,70 \\
\hline
\end{tabular}

Nota: As regressões do modelo são estimadas usando-se a metodologia de Fama e MacBeth (1974). 0 modelo foi estimado usando-se o método generalizado dos momentos. Através da correção dos erros, verificamos se a variância residual afeta o preço dos ativos ou a taxa de retorno esperada e, com base nos resultados, não existe uma indicação de que os ativos com variância residual maior que a média produzam taxa de retorno maior que a média num período futuro. Foram construídos cinco portfólios com cinco ações em cada um deles. O período testado vai de janeiro de 1994 até dezembro de 2002. Para o mercado argentino, o prêmio é representado pelo spread entre a taxa de juros sobre empréstimos ao setor privado e a taxa de juros básica da economia. Já o capital humano é representado pelo PIB argentino. A proxy de mercado será o índice Merval. A equação que está sendo estimada para o mercado argentino é a seguinte: $E\left[R_{i t}\right]=c_{0}+c_{\text {size }} \log \left(M E_{i}\right)+c_{\text {merval }} \beta+c_{\text {premio }} \beta+c_{\text {pib }} \beta$ 
ce ser mais eficaz para explicar a variação cross-sectional média dos retornos no mercado chileno.

$\mathrm{O}$ valor do $\mathrm{R} 2$ para o mercado brasileiro se manteve em $42,90 \%$ (valor muito parecido com o encontrado no CAPM estático) e o valor estimado para a variável $C_{\text {premio }}$, depois de corrigidos os erros, tornou-se significativamente diferente de zero, fatos estes que podem ser explicados pela não inclusão do PIB de mercado. Assim mesmo, o modelo condicional parece ser mais eficaz para explicar a variação cross-sectional média dos retornos nos mercado brasileiro e argentino. Análise semelhante à do mercado norte-americano.

A seguir, serão apresentadas as tabelas que mostram os resultados do modelo CAPM condicional com capital humano para os países analisados.

Os resultados presentes nas Tabelas 9, 10, 11 e 12 mostram que o valor estimado para o $\mathrm{C}_{\text {pib.mer }}$, usando-se a metodologia de Fama-MacBeth, não é significativamente diferente de zero. O t-value é de -0,21 e o R2 é de $11,76 \%$.

Quando a variável size é adicionada ao modelo, o

\section{Tabela 7 - CAPM condicional sem capital humano (Chile)}

\begin{tabular}{|l|c|c|c|c|c|c|}
\hline \multicolumn{1}{|c|}{ COEFICIENTES } & $\mathrm{C}_{0}$ & $\mathrm{C}_{\text {IPSE }}$ & $\mathrm{C}_{\text {PREMIO }}$ & $\mathrm{C}_{\text {PIB }}$ & $\mathrm{C}_{\text {SIIE }}$ & R-SQUARE \\
\hline Estimate & $-3,74$ & $-2,13$ & $-5,47$ & & \\
\hline t-value & $-1,18$ & $-0,86$ & $-0,90$ & & \\
\hline p-value & 0,10 & 0,08 & 0,00 & & \\
\hline Correção-t & $-1,24$ & $-1,02$ & $-1,12$ & & \\
\hline Correção-p & 0,20 & 0,10 & 0,00 & & \\
\hline Estimate & $-1,64$ & 1,93 & 1,23 & & \\
\hline t-value & $-1,32$ & 1,04 & 0,67 & & 40,24 \\
\hline p-value & 0,30 & 1,43 & 1,05 & & 0,13 & \\
\hline Correção-t & $-1,18$ & 0,89 & 0,53 & & 4,23 & \\
\hline Correção-p & 0,40 & 0,97 & 0,93 & & 0,08 & \\
\hline
\end{tabular}

Nota: As regressões do modelo são estimadas usando-se a metodologia de Fama e MacBeth (1974). 0 modelo foi estimado usando-se o método generalizado dos momentos. Através da correção dos erros, verificamos se a variância residual afeta o preço dos ativos ou a taxa de retorno esperada e, com base nos resultados, não existe uma indicação de que os ativos com variância residual maior que a média produzam taxa de retorno maior que a média num período futuro. Foram construídas cinco carteiras com cinco ações em cada uma delas. O período testado vai de janeiro de 1994 até dezembro de 2002.

$E\left[R_{i t}\right]=c_{0}+c_{\text {size }} \log \left(M E_{i}\right)+c_{\text {IPSEl }} \beta+c_{\text {premio }} \beta+c_{\text {pib }} \beta$

Tabela 8 - CAPM condicional sem capital humano (EUA)

\begin{tabular}{|l|c|c|c|c|c|c|}
\hline \multicolumn{1}{|c|}{ COEFICIENTES } & $\mathrm{C}_{0}$ & $\mathrm{C}_{\mathrm{W}}$ & $\mathrm{C}_{\text {PREMIO }}$ & $\mathrm{C}_{\text {PIB }}$ & $\mathrm{C}_{\text {SIIE }}$ & R-SQUARE \\
\hline Estimate & 0,81 & $-0,31$ & 0,36 & & \\
\hline t-value & 2,72 & $-0,87$ & 3,28 & & \\
\hline p-value & 0,66 & 38,45 & 0,1 & & \\
\hline Correção-t & 2,19 & $-0,7$ & 2,67 & & \\
\hline Correção-p & 2,87 & 48,43 & 0,75 & & \\
\hline Estimate & 1,77 & $-0,38$ & 0,16 & & \\
\hline t-value & 4,75 & $-1,1$ & 2,5 & & $-0,1$ & \\
\hline p-value & 0 & 27,17 & 1,26 & & $-1,93$ & \\
\hline Correção-t & 4,53 & $-1,05$ & 2,4 & & 5,35 & \\
\hline Correção-p & 0 & 29,53 & 1,66 & & $-1,84$ & \\
\hline
\end{tabular}


Tabela 9 - CAPM condicional com capital humano (Brasil)

\begin{tabular}{|l|c|c|c|c|c|c|}
\hline \multicolumn{1}{|c|}{ COEFICIENTES } & $\mathrm{C}_{0}$ & $\mathrm{C}_{\text {IBOV }}$ & $\mathrm{C}_{\text {PREMIO }}$ & $\mathrm{C}_{\text {PIB MER }}$ & $\mathrm{C}_{\text {SIZE }}$ & R-SQUARE \\
\hline Estimate & $-3,01$ & 4,67 & 3,89 & $-0,59$ & & 11,76 \\
\hline t-value & $-0,78$ & 0,12 & $-0,16$ & $-0,21$ & & \\
\hline p-value & 0,00 & 0,00 & 0,00 & 0,00 & & \\
\hline Correção-t & $-0,1$ & 0,01 & $-0,09$ & $-0,03$ & & \\
\hline Correção-p & 0,00 & 0,04 & 0,10 & 0,02 & & \\
\hline Estimate & $-0,85$ & 13,25 & 2,86 & $-1,28$ & 0,69 & 51,59 \\
\hline t-value & $-0,2$ & 0,33 & 0,18 & $-0,44$ & 2,62 & \\
\hline p-value & 0,00 & 0,00 & 0,00 & 0,00 & 0,00 & \\
\hline Correção-t & $-0,01$ & 0,02 & 0,10 & $-0,03$ & 0,21 & \\
\hline Correção-p & 0,04 & 0,02 & 0,85 & 0,01 & 0,00 & \\
\hline
\end{tabular}

Nota: As regressões do modelo são estimadas usando-se a metodologia de Fama e MacBeth (1974). 0 modelo foi estimado usando-se o método generalizado dos momentos. Através da correção dos erros, verificamos se a variância residual afeta o preço dos ativos ou a taxa de retorno esperada e, com base nos resultados, não existe uma indicação de que os ativos com variância residual maior que a média produzam taxa de retorno maior que a média num período futuro. Foram construídos sete portfólios com cinco ações em cada um deles. O período testado vai de janeiro de 1994 até dezembro de 2002. Para o mercado brasileiro, o prêmio é representado pelo spread entre a taxa da CETIP e a taxa SELIC. Já o capital humano é representado pelo PIB de mercado. A proxy de mercado será o Ibovespa. A equação que está sendo estimada para o mercado brasileiro é a seguinte:

$E\left[R_{i t}\right]=c_{0}+c_{\text {size }} \log \left(M E_{i}\right)+c_{i b o v} \beta+c_{\text {premio }} \beta+c_{\text {pib.mer }} \beta$

Tabela 10 - CAPM condicional com capital humano (Argentina)

\begin{tabular}{|l|l|l|l|l|l|l|}
\hline \multicolumn{1}{|c|}{ COEFICIENTES } & $\mathrm{C}_{0}$ & $\mathrm{C}_{\text {MERVAL }}$ & $\mathrm{C}_{\text {PREMIO }}$ & $\mathrm{C}_{\text {PIB }}$ & $\mathrm{C}_{\text {SIIE }}$ & R-SQUARE \\
\hline Estimate & 2,70 & $-2,45$ & 1,14 & 1,20 & & 25,70 \\
\hline t-value & 3,64 & $-2,70$ & 2,12 & 1,91 & & \\
\hline p-value & 0,10 & 14,97 & 0,00 & 3,70 & \\
\hline Correção-t & 2,14 & $-1,03$ & 1,38 & 2,90 & & \\
\hline Correção-p & 0,20 & 18,37 & 0,90 & 6,30 & & \\
\hline Estimate & 2,76 & $-2,30$ & 0,80 & 1,40 & $-1,09$ & 56,73 \\
\hline t-value & 2,13 & $-0,98$ & 4,70 & 3,45 & $-2,65$ & \\
\hline p-value & 0,20 & 11,48 & 0,37 & 4,67 & 8,90 & \\
\hline Correção-t & 3,70 & $-2,09$ & 5,89 & 3,94 & $-3,46$ & \\
\hline Correção-p & 0,30 & 13,14 & 0,76 & 6,53 & 12,30 & \\
\hline Correção-t: & 3,70 & $-2,09$ & 5,89 & 3,94 & $-3,46$ & \\
\hline Correção-p: & 0,30 & 13,14 & 0,76 & 6,53 & 12,30 \\
\hline
\end{tabular}

Nota: As regressões do modelo são estimadas usando-se a metodologia de Fama e MacBeth (1974). 0 modelo foi estimado usando-se o método generalizado dos momentos. Através da correção dos erros, verificamos se a variância residual afeta o preço dos ativos ou a taxa de retorno esperada e, com base nos resultados, não existe uma indicação de que os ativos com variância residual maior que a média produzam taxa de retorno maior que a média num período futuro. Foram construídos cinco portfólios com cinco ações em cada um deles. O período testado vai de janeiro de 1994 até dezembro de 2002. Para o mercado argentino, o prêmio é representado pelo spread entre a taxa de juros sobre empréstimos ao setor privado e a taxa de juros básica da economia. Já o capital humano é representado pelo PIB argentino. A proxy de mercado será o índice Merval. A equação que está sendo estimada para o mercado argentino é a seguinte:

$E\left[R_{i t}\right]=c_{0}+c_{\text {size }} \log \left(M E_{i}\right)+c_{\text {merval }} \beta+c_{\text {premio }} \beta+c_{\text {pib }} \beta$ 
t-value para o mercado argentino da variável $\mathrm{C}_{\text {size }}$ é de $-2,65$ com p-value de $8,90 \%$ e $R 2$ de $56,73 \%$. A variável size não consegue explicar o que não havia sido explicado no modelo com a inclusão do controle dos erros da amostra. Quando os erros são corrigidos, o $p$-value para o $C_{\text {size }}$ torna-se ainda maior, reforçando essas conclusões muito semelhantes às do mercado norte-americano.

Já no mercado brasileiro, quando se introduz o modelo de correção de erros, o t-value para $C_{\text {pib.mer }}$ vai para -0,03, o $p$-value vai para 0,02 e o coeficiente $C_{\text {premio }}$ torna-se sig- nificante. Quando a variável size é adicionada ao modelo,

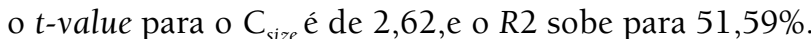

Em relação ao mercado chileno, conclui-se que o CAPM condicional com a inclusão do PIB chileno parece aproximar-se dos resultados obtidos no mercado brasileiro.

O CAPM condicional com a inclusão do PIB de mercado brasileiro parece aproximar-se dos resultados obtidos nos mercados norte-americano e argentino. Apesar das variáveis $C_{\text {premio }}$ e $C_{\text {pibmer }}$ se tornarem significativamente

Tabela 11 - CAPM condicional com capital humano (Chile)

\begin{tabular}{|l|c|c|c|c|c|c|}
\hline \multicolumn{1}{|c|}{ COEFICIENTES } & $\mathrm{C}_{0}$ & $\mathrm{C}_{\text {IPSE }}$ & $\mathrm{C}_{\text {PREMIO }}$ & $\mathrm{C}_{\text {PIB }}$ & $\mathrm{C}_{\text {SIIE }}$ & R-SQUARE \\
\hline Estimate & $-4,80$ & 6,32 & 5,34 & $-1,25$ & & 10,10 \\
\hline t-value & $-1,97$ & 1,44 & $-1,21$ & $-1,03$ & \\
\hline p-value & 0,43 & 0,10 & 0 & 0,40 & \\
\hline Correção-t & $-1,38$ & 1,13 & $-0,97$ & $-0,87$ & \\
\hline Correção-p & 0,67 & 0,08 & 0,00 & 0,97 & & \\
\hline Estimate & $-1,74$ & 9,25 & 3,47 & $-2,75$ & 1,32 & 49,20 \\
\hline t-value & $-0,90$ & 0,95 & 1,06 & $-1,54$ & 3,36 & \\
\hline p-value & 0,00 & 0,00 & 0,10 & 0,12 & 0 & \\
\hline Correção-t & $-0,34$ & 0,47 & 0,93 & $-1,21$ & 1,92 & \\
\hline Correção-p & 0,00 & 0,00 & 0,71 & 0,45 & 0 & \\
\hline
\end{tabular}

Nota: As regressões do modelo são estimadas usando-se a metodologia de Fama e MacBeth (1974). 0 modelo foi estimado usando-se o método generalizado dos momentos. Através da correção dos erros, verificamos se a variância residual afeta o preço dos ativos ou a taxa de retorno esperada e, com base nos resultados, não existe uma indicação de que os ativos com variância residual maior que a média produzam taxa de retorno maior que a média num período futuro. Foram construídos cinco carteiras com cinco ações em cada uma delas. O período testado vai de janeiro de 1994 até dezembro de 2002.

$E\left[R_{i t}\right]=c_{0}+c_{\text {size }} \log \left(M E_{i}\right)+c_{\text {IPSEl }} \beta+c_{\text {premio }} \beta+c_{\text {pib }} \beta$

Tabela 12 - CAPM condicional com capital humano (EUA)

\begin{tabular}{|l|c|c|c|c|c|c|}
\hline \multicolumn{1}{|c|}{ COEFICIENTES } & $\mathrm{C}_{0}$ & $\mathrm{C}_{\mathrm{W}}$ & $\mathrm{C}_{\text {PREM }}$ & $\mathrm{C}_{\text {LABOR }}$ & $\mathrm{C}_{\text {SIIE }}$ & R-SQUARE \\
\hline Estimate & 1,24 & $-0,4$ & 0,34 & 0,22 & & 55,21 \\
\hline t-value & 5,51 & $-1,18$ & 3,31 & 2,31 & & \\
\hline p-value & 0 & 23,76 & 0,09 & 2,07 & & \\
\hline Correção-t & 4,1 & $-0,88$ & 2,48 & 1,73 & & \\
\hline Correção-p & 0 & 37,99 & 1,31 & 8,44 & & \\
\hline Estimate & 1,7 & $-0,4$ & 0,2 & 0,1 & $-0,07$ & 64,73 \\
\hline t-value & 4,61 & $-1,18$ & 3 & 2,09 & $-1,45$ & \\
\hline p-value & 0 & 23,98 & 0,27 & 3,62 & 14,74 & \\
\hline Correção-t & 4,14 & $-1,06$ & 2,72 & 1,89 & $-1,3$ & \\
\hline Correção-p & 0 & 29,07 & 0,66 & 5,87 & 19,29 & \\
\hline
\end{tabular}


diferentes de zero após a correção dos erros, a consistência do modelo não parece ter sido abalada.

A seguir, serão apresentadas as tabelas mostrando os resultados do modelo CAPM estático com capital humano para os países analisados.

Os resultados presentes nas Tabelas 13 e 14 acima

\section{Tabela 13 - CAPM estático com capital humano (Brasil)}

\begin{tabular}{|c|c|c|c|c|c|c|}
\hline COEFICIENTES & $\mathrm{C}_{0}$ & $\mathrm{C}_{\mathrm{BBOV}}$ & $\mathrm{C}_{\text {PREMIO }}$ & $\mathrm{C}_{\text {PIB MER }}$ & $\mathrm{C}_{\text {SIZE }}$ & R-SQUARE \\
\hline Estimate & $-2,83$ & 6,91 & & $-0,62$ & & 10,75 \\
\hline t-value & $-0,79$ & 0,30 & & $-0,23$ & & \\
\hline$p$-value & 0,00 & 0,00 & & 0,00 & & \\
\hline Correção-t & $-0,09$ & 0,03 & & $-0,03$ & & \\
\hline Correção-p & 0,00 & 0,01 & & 0,04 & & \\
\hline Estimate & $-0,98$ & 11,78 & & $-1,25$ & 0,68 & 50,83 \\
\hline t-value & $-0,26$ & 0,49 & & $-0,46$ & 3,29 & \\
\hline$p$-value & 0,00 & 0,00 & & 0,00 & 0,00 & \\
\hline Correção-t & $-0,02$ & 0,03 & & $-0,03$ & 0,28 & \\
\hline Correção-p & 0,02 & 0,01 & & 0,05 & 0,00 & \\
\hline
\end{tabular}

Nota: As regressões do modelo são estimadas usando-se a metodologia de Fama e MacBeth (1974). 0 modelo foi estimado usando-se o método generalizado dos momentos. Através da correção dos erros, verificamos se a variância residual afeta o preço dos ativos ou a taxa de retorno esperada e, com base nos resultados, não existe uma indicação de que os ativos com variância residual maior que a média produzam taxa de retorno maior que a média num período futuro. Foram construídos sete portfólios com cinco ações em cada um deles. O período testado vai de janeiro de 1994 até dezembro de 2002. Para o mercado brasileiro, o prêmio é representado pelo spread entre a taxa da CETIP e a taxa SELIC. Já o capital humano é representado pelo PIB de mercado. A proxy de mercado será o Ibovespa. A equação que está sendo estimada para o mercado brasileiro é a seguinte:

$E\left[R_{i t}\right]=c_{0}+c_{\text {size }} \log \left(M E_{i}\right)+c_{i b o v} \beta+c_{\text {premio }} \beta+c_{\text {pib.mer }} \beta$

Tabela 14 - CAPM estático com capital humano (Argentina)

\begin{tabular}{|l|l|l|l|l|l|l|}
\hline \multicolumn{1}{|c|}{ COEFICIENTES } & $\mathrm{C}_{0}$ & $\mathrm{C}_{\text {MERVAL }}$ & $\mathrm{C}_{\text {PREMIO }}$ & $\mathrm{C}_{\text {PIB }}$ & $\mathrm{C}_{\text {SIIE }}$ & R-SQUARE \\
\hline Estimate & 2,54 & $-1,28$ & & 1,35 & & 16,50 \\
\hline t-value & 2,47 & $-1,45$ & & 3,78 & \\
\hline p-value & 0,00 & 25,56 & & 2,14 & \\
\hline Correção-t & 3,83 & $-1,12$ & 2,83 & & \\
\hline Correção-p & 0,00 & 28,30 & 6,17 & & \\
\hline Estimate & 3,89 & $-1,24$ & 1,43 & $-1,12$ & 53,18 \\
\hline t-value & 7,64 & $-2,37$ & 2,23 & $-3,46$ & \\
\hline p-value & 0,00 & 16,48 & 11,35 & 4,76 & \\
\hline Correção-t & 8,45 & $-1,85$ & & 2,21 & $-3,37$ & \\
\hline Correção-p & 0,00 & 17,60 & 12,02 & 4,50 & \\
\hline
\end{tabular}

Nota: As regressões do modelo são estimadas usando-se a metodologia de Fama e MacBeth (1974). 0 modelo foi estimado usando-se o método generalizado dos momentos. Através da correção dos erros, verificamos se a variância residual afeta o preço dos ativos ou a taxa de retorno esperada e, com base nos resultados, não existe uma indicação de que os ativos com variância residual maior que a média produzam taxa de retorno maior que a média num período futuro. Foram construídos cinco portfólios com cinco ações em cada um deles. O período testado vai de janeiro de 1994 até dezembro de 2002. Para o mercado argentino, o prêmio é representado pelo spread entre a taxa de juros sobre empréstimos ao setor privado e a taxa de juros básica da economia. Já o capital humano é representado pelo PIB argentino. A proxy de mercado será o índice Merval. A equação que está sendo estimada para o mercado argentino é a seguinte:

$E\left[R_{i t}\right]=c_{0}+c_{\text {size }} \log \left(M E_{i}\right)+c_{\text {merval }} \beta+c_{\text {premio }} \beta+c_{\text {pib }} \beta$ 
mostram que o valor estimado para o $C_{\text {pibmer }}$, usando-se a metodologia de Fama-MacBeth, não é significativamente diferente de zero. O t-value é de -0,23. O R2 é de apenas $10,75 \%$. Após a correção dos erros, conclui-se que o $C_{\text {pibmer }}$ se torna significativamente diferente de zero, ao contrário do mercado norte-americano.

Quando introduzirmos a variável size, o t-value é de 3,29 e o $R 2$ sobe para $50,83 \%$. Apesar do elevado R2, o modelo não é consistente. É necessário permitir que o beta varie no tempo para explicar o retorno cross-sectional esperado dos mercados.
Quando introduzirmos a variável size no mercado argentino, o t-value é de -3,46 com $p$-value de 4,76. O R2 sobe para $53,18 \%$. A introdução da variável size parece não conseguir explicar o aumento verificado no $R 2$. Apesar do aumento do $R 2$ e de os resultados serem coerentes com a literatura, o modelo não parece ser consistente com os dados e, assim, recomenda-se a introdução de novos parâmetros.

Os resultados presentes na Tabela 15 mostram que o valor estimado para o $\mathrm{C}_{\mathrm{PIB}}$, usando-se a metodologia de Fama-MacBeth, não é significativamente diferente

\section{Tabela 15 - CAPM estático com capital humano (Chile)}

\begin{tabular}{|l|c|c|c|c|c|c|}
\hline \multicolumn{1}{|c|}{ COEFICIENTES } & $\mathrm{C}_{0}$ & $\mathrm{C}_{\text {IPSE }}$ & $\mathrm{C}_{\text {PREMIO }}$ & $\mathrm{C}_{\text {PIB }}$ & $\mathrm{C}_{\text {SIIE }}$ & R-SQUARE \\
\hline Estimate & $-3,70$ & 7,54 & & $-1,45$ & & 11,13 \\
\hline t-value & $-1,67$ & 1,38 & & $-1,07$ & \\
\hline p-value & 0,10 & 0,08 & & 0,17 & \\
\hline Correção-t & $-1,13$ & 0,97 & $-0,85$ & \\
\hline Correção-p & 0,18 & 0,12 & & & \\
\hline Estimate & $-1,76$ & 8,93 & & $-2,38$ & 1,46 & 51,45 \\
\hline t-value & $-1,04$ & 1,32 & $-1,18$ & 4,74 & \\
\hline p-value & 0,19 & 0,00 & 0,25 & 0,00 & \\
\hline Correção-t & $-0,76$ & 1,02 & $-0,93$ & 3,14 & \\
\hline Correção-p & 0,23 & 0 & 0,32 & 0,00 & \\
\hline
\end{tabular}

Nota: As regressões do modelo são estimadas usando-se a metodologia de Fama e MacBeth (1974). O modelo foi estimado usando-se o método generalizado dos momentos. Através da correção dos erros, verificamos se a variância residual afeta o preço dos ativos ou a taxa de retorno esperada e, com base nos resultados, não existe uma indicação de que os ativos com variância residual maior que a média produzam taxa de retorno maior que a média num período futuro. Foram construídas cinco carteiras com cinco ações em cada um deles. O período testado vai de janeiro de 1994 até dezembro de 2002.

$E\left[R_{i t}\right]=c_{0}+c_{\text {size }} \log \left(M E_{i}\right)+c_{\text {IPSEI }} \beta+c_{\text {premio }} \beta+c_{\text {pib }} \beta$

Tabela 16 - CAPM estático com capital humano (EUA)

\begin{tabular}{|c|c|c|c|c|c|c|}
\hline COEFICIENTES & $\mathrm{C}_{0}$ & $\mathrm{C}_{\mathrm{w}}$ & $\mathrm{C}_{\text {PREM }}$ & $\mathrm{C}_{\mathrm{LABOR}}$ & $\mathrm{C}_{\text {SIZE }}$ & R-SQUARE \\
\hline Estimate & 1,67 & $-0,22$ & & 0,23 & & 30,46 \\
\hline t-value & 6,91 & $-0,63$ & & 2,37 & & \\
\hline $\mathrm{p}$-value & 0 & 53,19 & & 1,77 & & \\
\hline Correção-t & 5,71 & $-0,52$ & & 1,97 & & \\
\hline Correção-p & 0 & 60,49 & & 4,87 & & \\
\hline Estimate & 2,09 & $-0,32$ & & 0,05 & $-0,1$ & 58,55 \\
\hline t-value & 5,8 & $-0,96$ & & 1,22 & $-2,15$ & \\
\hline $\mathrm{p}$-value & 0 & 33,78 & & 22,29 & 3,19 & \\
\hline Correção-t & 5,7 & $-0,95$ & & 1,2 & $-2,11$ & \\
\hline Correção-p & 0 & 34,46 & & 22,93 & 3,48 & \\
\hline
\end{tabular}


de zero. O t-value é de -1,07. O R2 é de apenas 11,13\%. Após a correção dos erros, conclui-se que o $C_{\mathrm{PIB}}$ se torna significativamente diferente de zero, ao contrário dos mercados norte-americano e argentino, e semelhante ao mercado brasileiro.

\section{CONCLUSÕES}

O CAPM estático, sem a inclusão da variável capital humano, parece não explicar satisfatoriamente o retorno cross-sectional esperado dos mercados analisados.

Após a inclusão da variável size, os $R 2$ de todos os modelos apresentaram uma abrupta mudança. Apesar desse fato e de os resultados encontrados serem coerentes com a literatura, conclui-se que o modelo para os países analisados parece inconsistente por não apresentar nenhuma mudança nos parâmetros ao longo do tempo.

O modelo estático parece não representar satisfatoriamente a realidade de cada uma das economias analisadas. Primeiramente, porque sabemos que o ciclo de negócios é dinâmico em qualquer economia e o modelo analisado acima não contempla esse fato. Segundo, porque a proxy de mercado, por si só, não é suficiente para representar uma economia, seja ela qual for.

Esse modelo deve ser aperfeiçoado com a inclusão de novas variáveis que consigam representar bem qualquer que seja o mercado.

Entretanto, não temos motivos consistentes para, simplesmente, desprezar o CAPM estático, pois ele é capaz de explicar o mercado para determinado lapso de tempo.

Diferentemente dos mercados norte-americano e argentino, os mercados brasileiro e chileno têm relação crescente entre os retornos médios das carteiras e o tamanho, tendo a carteira de tamanho maior retorno substancialmente mais alto.

Em relação ao CAPM condicional, sem a inclusão da variável capital humano, podemos constatar que, no caso brasileiro, o valor estimado para o $C_{\text {premio }}$ não é significativamente diferente de zero, enquanto nos casos norte-americano e argentino é significativamente diferente de zero.

Entretanto, quando se introduz o modelo de correção dos erros, a variável $C_{\text {premio }}$ se torna significativamente diferente de zero para os casos brasileiro e chileno. Nos casos norte-americano e argentino, mesmo após a adoção do modelo de correção de erros, a variável $C_{\text {premio }}$ se mantém significativamente diferente de zero. Isto significa que o prêmio de risco influencia demasiadamente o mercado analisado.

Quando a variável size é adicionada ao modelo, o R2 sobe proporcionalmente para os dados brasileiros e chile- nos. Para os dados norte-americanos e argentinos, quando é incluída a variável size, o $R 2$ sobe de forma menos significativa.

Quando a variável size é adicionada aos modelos, seus $R 2$ sofrem um aumento considerável, apesar de a variável size apresentar alguns efeitos no modelo. Isto quer dizer que o CAPM condicional, mesmo sem a inclusão do capital humano, consegue explicar de forma mais eficaz a variação cross-sectional média dos retornos dos portfólios analisados. Tal resultado é encontrado porque a variável size ou efeito tamanho influencia muito os mercados brasileiro e chileno.

Em relação ao modelo condicional, podemos concluir que, sem dúvida, o poder de explicação do modelo aumenta muito, seja para qualquer um dos casos analisados.

O modelo parece conseguir captar o efeito dinâmico da economia. Introduzindo-se a variável size, os modelos têm um aumento considerável em seus $R 2$, mas se nota que essa variável parece ser mais significativa no mercado brasileiro, devido, provavelmente, às diferenças encontradas nas composições de ações desse mercado.

Finalmente, encontram-se evidências de que o CAPM condicional de Jagannathan e Wang (1996) para o mercado norte-americano é perfeitamente aplicável aos mercados brasileiro, argentino e chileno. Os resultados encontrados acima para os mercados nos permitem diferenciar e também identificar algumas características próprias de cada um deles. Isto se torna uma importante ferramenta para o investidor potencial desses países, já que são encontradas diferenças no comportamento dos mercados analisados.

Embora o modelo condicional tenha um desempenho substancialmente melhor do que o modelo estático, recomendam-se cuidados na interpretação dos resultados do CAPM condicional pelas seguintes razões: (1) o modelo apresentado de betas variando no tempo é ainda um tanto simples. No mundo dinâmico, investidores podem realizar hedge, contra a variedade de riscos que não existem na economia estática; (2) a análise do CAPM condicional é fundamental, pois a especificação do CAPM não condicional pode levar a falsas conclusões em relação à validade do modelo (alguns artigos mostram que o CAPM condicional não é válido quando estamos analisando períodos de tempo muito longos); (3) o número de eventos determinísticos que ocorrem com frequência mensal e anual. Espera-se que esses eventos influenciem o comportamento dos modelos de precificação de ativos naqueles períodos. Para eventos que estão fora da ação dos modelos de precificação de ativos semelhantes ao CAPM, uma das estratégias deveria ser a de estudar o desempe- 
nho de modelos usando-se dados anuais para um longo período de tempo, como em Jagannathan e Wang (1993) e Kothari, Shanken e Sloan (1995).

\section{REFERÊNCIAS}

BANSAL, R; VISWANAYHAN, S. No arbitrage pricing: a new approach. Journal of Finance, v. 48, n. 4, p. 1231-1262, 1993

BERK, J. B. A critique of size-related anomalies. Review of Financial Studies, v. 8 , n. 2, p. $275-286,1995$.

BLACK, FISCHER. Beta and return. Journal of Portfolio Management, v. 20, n. 1, p. 8-18, 1993.

BLACK, FISCHER. Capital market equilibrium with restricted borrowing. Journal of Business, v.45, n. 3, p. 444-455, 1972.

BLACK, FISCHER; JENSEN, M. C. Myron Scholes. The Capital Asset Pricing Model: Some Empirical Tests, in M. Jensen ed., Studies in the Theory of Capital Markets, p. 79-121, New York: Praeger Publishers, 1972.

BODURTHA JR, J. N; MARK, N. C. Testing the CAPM with time-varying risks and returns. Journal of Finance, v.46, n. 4, p. 1485-1505, 1991.

BONOMO, Marco. Finanças aplicadas ao Brasil. São Paulo: FGV Editora, 2002. p.12-15.

COSTA JÚNIOR, N. C. A. Será que beta ainda é válido para explicar as variações nas rentabilidades médias das ações? In: ENCONTRO ANUAL DA ASSOCIAÇÃO NACIONAL DOS PROGRAMAS DE PÓS-GRADUAÇÃO EM ADMINISTRAÇÃO, 20, 1996, Petrópolis. Anais... Petrópolis: ANPAD, 1996. p. 335-348.

FAMA, E. F; French, K. R. Common risk factors in the returns on bonds and stocks. Journal of Financial Economics, v. 33, n. 1, p. 3-56, 1993.

FAMA, E. F; French, K. R. The cross-section of expected stock returns Journal of Finance, v. 47, n. 2, p. 427-466, 1992.

FAMA, E. F; MacBeth, J. D. Risk, return and equilibrium. Empirical tests. Journal of Political Economy, v. 81, n. 3, p. 607-636, 1973.

FAMA, E. F; MacBeth, J. D. Tests of the multiperiod two-parameter model. Journal of Financial Economics, v. 1, n. 1, p. 43-66, 1974

FERSON, W. E; HARVEY, C. R. The variation of economic risk premiums. Journal of Political Economy, v. 99, n. 2, p. 385-415, 1991.

FERSON, W. E; HARVEY, C. R. The risk and predictability of international equity returns. Review of Financial Studies, v. 6, n. 3, p. 527-566, 1993.
FERSON, W. E; HARVEY, C. R. Conditioning Variables and the Cross Section of Stock Returns. The Journal of Finance, v. 54, n. 4, p. 13251360, 1999.

HAUGEN, R. A. Modern investment theory. New Jersey: Prentice-Hall, 1986.

JAGANNATHAN, R; WANG, Z. The CAPM is alive and well. Staff Report 165, Federal Reserve Bank of Minneapolis. 1993.

JAGANNATHAN, R; WANG, Z. The conditional CAPM and the cross-section of expected returns. Journal of Finance, v.51, n. 1, p. 3-53, Mar. 1996

KEIM, DONALD B., and STAMBAUGH, Robert F. Predicting Returns in the Stock and Bond Markets. Journal Financial Econ., v. 17, n. 2, p.357390,December 1986

KOTHARI, S. P; Shanken, J; SLOAN, R. G.. Another look at the cross-section of expected stock returns. Journal of Finance, v.50, n. 1, p. 185-224

LINTNER, J. The valuation of risk assets and the selection of risk investments in stock portfolio and capital budgets. Review of Economics and Statistics, v. 47, n. 1, p. 13-37, 1965

MAYERS, D. Non marketable assets and capital market equilibrium under uncertainty. In: Jensen, M. C. Studies in the theory of capital markets, New York: Praeger Publishers, p. 223-248, 1972.

MERTON, R. C. An intertemporal capital asset pricing model. Econometrica. v. 41, n. 5, p. 867-887, 1973.

MOSSIN, J. Equilibrium in a capital asset market. Econometrica, v. 34, n. 4, p. 768-783, Oct. 1966.

RIBENBOIM, G. Testes de versões do modelo CAPM no Brasil. In BONOMO, Marco. Finanças aplicadas ao Brasil. São Paulo: FGV Editora, 2002. p. $18-40$

ROLL, R. A critique of the asset pricing theory's tests. Journal of Financial Economics, v. 4, n. 2, p. 129-176, 1977.

SHARPE, W. F. Capital asset prices: a theory of market equilibrium under conditions of risk. Journal of Finance, v. 19, n. 3, p. 425-442, 1964.

WILLIAM BREEN, LAWRENCE R. GLOSTEN, and RAVI JAGANNATHAN Economic Significance of Predictable Variations in Stock Index Returns. The Journal of finance, v. 44, n. 5, p. 1177-1189, December 1989. 